\title{
Instant preheating mechanism and ultrahigh energy cosmic rays
}

\author{
A. H. Campos, ${ }^{1, *}$ J. M. F. Maia, ${ }^{2, \dagger}$ and R. Rosenfeld ${ }^{3, \dagger}$ \\ ${ }^{1}$ Instituto de Física-Universidade de São Paulo, Departamento de Física Nuclear - CP 66318, São Paulo, SP, Brazil \\ ${ }^{2}$ Conselho Nacional de Pesquisa e Desenvolvimento-COCEX, SEPN 509, Bl. A, Ed. Nazir I, 70750-901, Brasília, DF, Brazil \\ ${ }^{3}$ Instituto de Física Teórica-Universidade Estadual Paulista, Rua Pamplona, 145 - 01405-900, São Paulo, SP, Brazil
}

(Received 1 February 2004; published 23 July 2004)

\begin{abstract}
Top-down models assume that the still unexplained ultrahigh energy cosmic rays (UHECR's) are the decay products of superheavy particles. Such particles may have been produced by one of the post-inflationary reheating mechanisms and may account for a fraction of the cold dark matter. In this paper, we assess the phenomenological applicability of the simplest instant preheating framework not to describe a reheating process, but as a mechanism to generate relic supermassive particles as possible sources of UHECR's. We use cosmic ray flux and cold dark matter observational data to constrain the parameters of the model.
\end{abstract}

DOI: 10.1103/PhysRevD.70.023003

PACS number(s): 98.70.Sa, 98.80.Cq

\section{INTRODUCTION}

The possible observation of ultrahigh energy cosmic ray (UHECR) events with primary energies above $10^{20} \mathrm{eV}[1]$ constitute one of the most intriguing puzzles in astroparticle physics (see, for example, Ref. [2]), since their origin and composition are not yet understood. The usual bottom-up scenarios in which particles should be accelerated by astrophysical objects do not seem to provide a convincing solution to the puzzle. The arrival direction of the primary particles should point to their sources because at such energies the intergalactic magnetic field does not deviate their direction of propagation. However, the clustering of UHECR events observed in the available data is not statistically significant and therefore there is no evidence that they arise from point sources [3]. In addition, it would be necessary to overestimate several parameters of such sources and their acceleration regions in order to reach, marginally, the required energies [4].

The problem concerning cosmic ray sources is related to the necessity that they must be located in our neighborhood, since particles propagating at high energies suffer a rapid degradation of their energy. For protons or nuclei as primaries, interactions with the cosmic microwave background should cause a loss of their energy due to photopion production. Such an effect should result in a discontinuity in the cosmic ray spectrum for energies above $\sim 4 \times 10^{19} \mathrm{eV}$, the so-called Greisen-Zatsepin-Kuzmin (GZK) cutoff [5]. If the Auger Observatory data confirms that this feature is not observed, it can be shown [6] that protons must have travelled less than $\sim 100 \mathrm{Mpc}$ (attenuation length) in order to arrive at Earth with energies larger than $10^{19} \mathrm{eV}$. The attenuation length for photons depends on their initial energy and it is less than $100 \mathrm{Mpc}$ for energies between $10^{12} \mathrm{eV}$ to $10^{22} \mathrm{eV}$ [7]. Since neutrinos have a very small cross section with nucleons within the standard model, it seems difficult that they could produce air showers in our atmosphere unless

\footnotetext{
*Electronic address: hcampos@dfn.if.usp.br

†Electronic address: jmaia@cnpq.br

‡Electronic address: rosenfel@ift.unesp.br
}

they had a yet unknown interaction [8].

In order to overcome such difficulties, another class of models has been proposed [9]. The primary particle would not acquire kinetic energy continuously inside an accelerating region ("bottom-up" mechanism) as initially thought. Instead, the highly energetic cosmic rays would be originated by the decay products of superheavy particles of cosmological origin ("top-down" mechanism). For simplicity, we will consider that such particles have masses close to the grand unified theory (GUT) scale and would decay into known particles, as quarks and leptons that evolve following the QCD model [10]. The quarks hadronize, producing a small fraction of nucleons and pions that in turn decay into photons, neutrinos, and electrons and their corresponding antiparticles. Therefore, from the decay of such a supermassive particle it is possible to produce energetic photons, neutrinos, and leptons, together with a small percentage of nucleons. Depending on which kind of particle is the primary one, different attenuation lengths can be obtained, so that one can establish the minimum distances at which the supermassive particles sources should be located.

There are different exotic candidates to play the main role in top-down scenarios, such as decaying topological defects [11] or evaporating primordial black holes [12]. The simplest top-down models (at least from the particle physics point of view) involve supermassive metastable particles sometimes called wimpzillas [13]. Due to their colossal masses, such particles were presumably produced during the postinflationary epoch and could contribute to a part or to the whole of the dark matter that accounts for about $30 \%$ of the energy density of the Universe. In order to explain the theoretically estimated UHECR fluxes [14], such particles must be decaying now and have to be located in our neighborhood, which is expected, assuming they are concentrated in our galactic halo.

In this work we study the possibility of producing wimpzillas in the post-inflationary process called instant preheating, suggested by Felder, Kofman, and Linde (FKL), originally proposed as an alternative preheating mechanism [15]. This process seems to be essential for particle production in models of quintessential inflation $[16,17]$.

In such a scenario, scalar particles $\chi$ are nonperturbatively 
produced from the coherent oscillations of the inflaton $\phi$, have their masses "boosted" due to their coupling to the field $\phi$, and subsequently decay into supermassive metastable fermions $\psi$. The idea of examining stable supermassive particles in this context was addressed by Felder et al., but its consequences either as dark matter or as cosmic rays primaries were not calculated in detail.

More specifically, there is a relation between the density parameter of these particles and their lifetime. If $\psi$ particles compose the whole of the dark matter $\left(\Omega_{\psi} \equiv m_{\psi} n_{\psi} / \rho_{\text {crit }}\right.$ $\sim 0.3$ ) [18], a maximum lifetime limit will be found. On the other hand, a lower limit on the abundance of such particles can be obtained if lifetimes are constrained to be larger than the age of the Universe $\left(\tau_{\psi} \geqslant 10^{10} \mathrm{yr}\right)[19]$. As will be shown later, such limits impose severe constraints on the parameters of the FKL mechanism.

This paper is organized as follows. In the next section we review some features of the nonperturbative processes more directly related to the production of massive scalar particles. In Sec. III we perform a detailed calculation of superheavy $\psi$ particle production, extending the previous results in Ref. [15]. In Sec. IV we discuss our main results for produced particles considered as dark matter in our current Universe and present the parameter space for this model, which is in accordance with cosmological data. In the last section we present a summary of our main results and discuss their consequences.

\section{PRODUCTION OF $\chi$ PARTICLES}

After inflation, matter had to be (re)created, since the Universe became empty. In the case of chaotic inflation, the scenario considered here [20], particle production may occur during the quasiperiodic evolution phase of the inflaton field. To study such a period we assume the Lagrangian:

$$
L=\frac{1}{2} \partial_{\mu} \phi \partial^{\mu} \phi-V(\phi)+\frac{1}{2} \partial_{\mu} \chi \partial^{\mu} \chi-\frac{1}{2} m_{\chi}^{2} \chi^{2}-\frac{1}{2} g^{2} \phi^{2} \chi^{2} .
$$

The inflaton field $\phi$ produces quantum scalars bosons $\chi$ with bare masses $m_{\chi}$ through the interaction term $-g^{2} \phi^{2} \chi^{2} / 2$. For simplicity, we will limit our analyses to the quadratic potential $V(\phi)=m_{\phi}^{2} \phi^{2} / 2$ (the simplest chaotic inflation model) with $m_{\phi} \approx 10^{-6} M_{P l}$, as required by microwave background anisotropy measurements.

During inflation we can neglect the contribution of the $\chi$ field and the equation of motion for the $\phi$ field is given by

$$
\ddot{\phi}+3 H \dot{\phi}+m_{\phi}^{2} \phi=0
$$

where $H=\dot{a} / a$ is the Hubble parameter and obeys the Friedmann equation:

$$
H^{2}=\frac{8 \pi}{3 M_{P l}^{2}}\left(\frac{\dot{\phi}^{2}}{2}+\frac{m_{\phi}^{2} \phi^{2}}{2}\right)
$$

As far as the slow roll conditions are valid $[\ddot{\phi} \ll 3 H \dot{\phi}$, $\left.\dot{\phi}^{2} / 2 \ll V(\phi), \phi \approx M_{P l} / 3\right]$, the Universe is in an inflationary phase. Right after inflation, the $\phi$ field oscillates about the minimum of its potential, with decreasing amplitude due to the damping term $3 H \dot{\phi}$, and the solution of the above equation becomes

$$
\phi(t) \approx \frac{M_{P l}}{3} \frac{\sin \left(m_{\phi} t\right)}{m_{\phi} t} .
$$

The $\phi$ field may produce $\chi$ particles during this oscillating phase due to nonperturbative effects [21,22], provided the coupling constants have appropriate values. As $\chi$ particles are bosons, such a process may lead to an explosive particle production through parametric resonance of the $\chi$ field [23]. To illustrate this behavior, we consider the quantum nature of $\chi$ :

$$
\hat{\chi}(t, \vec{x})=\frac{1}{(2 \pi)^{3 / 2}} \int d^{3} k\left(\hat{a}_{k} \chi_{k}(t) e^{-i \vec{k} \cdot \vec{x}}+\hat{a}_{k}^{\dagger} \chi_{k}^{*}(t) e^{i \vec{k} \cdot \vec{x}}\right),
$$

where $\hat{a}_{k}$ and $\hat{a}_{k}^{\dagger}$ are the creation and annihilation operators, respectively. The equations of motion for the $k$ modes of the $\chi$ eigenfunctions are given by

$$
\ddot{\chi}_{k}(t)+3 H \dot{\chi}_{k}(t)+\left(\frac{k^{2}}{a^{2}(t)}+m_{\chi}^{2}+g^{2} \phi^{2}(t)\right) \chi_{k}(t)=0 .
$$

Rewriting the above equation in terms of a more convenient variable $X_{k} \equiv a^{3 / 2} \chi_{k}$, we obtain

$$
\ddot{X}_{k}+\left(\frac{k^{2}}{a^{2}}+m_{\chi}^{2}+g^{2} \phi^{2}\right) X_{k}=0,
$$

where we used the fact that, for the quadratic chaotic potential, the inflaton coherent oscillations redshift as nonrelativistic matter. Note that this is an oscillator equation with a variable frequency

$$
\omega_{k}(t)=\sqrt{\frac{k^{2}}{a^{2}(t)}+m_{\chi}^{2}+g^{2} \phi^{2}(t)} .
$$

The effective mass of $\chi$ is defined as

$$
m_{\chi}^{\mathrm{eff}}(t)=\sqrt{m_{\chi}^{2}+g^{2} \phi^{2}(t)} .
$$

Depending on the values of the parameters, the time variation of $\omega_{k}$ will not be adiabatic. The loss of adiabaticity takes place when $\phi$ field passes through the minimum of its potential, the region where $\omega_{k}(t)$ changes more rapidly. In such a case, there will be an inequivalence between the $X$ vacua defined before and after the passage of the inflaton through the minimum, which can be interpreted as production of $\chi$ particles [23]. This particle production process has been considered mainly for preheating proposals, since it happens before the usual perturbative reheating. Alternatively, such a coupling between $\phi$ and $\chi$ can be used in models with production of heavy metastable particles in the early Universe. 
In such a case, the produced particles may have masses larger than the inflaton mass [24]. In what follows, we work on the latter approach and consider $g$ and $m_{\chi}$ as free parameters to be estimated from the available cosmological data.

\section{THE FELDER-KOFMAN-LINDE MECHANISM}

Felder et al. [15] noticed that the preheating could be efficient even in a single $\phi$ field oscillation if the $\chi$ particles produced at the minimum of $V(\phi)$ were allowed to decay perturbatively into $\psi$ fermions when $\phi$ reached the maximum of its potential energy. The decay of such fermions into other particles and their subsequent thermalization could complete the reheating process without the necessity of parametric resonance. The authors also suggested the possibility of using such a mechanism to produce heavy particles in order to explain cosmic rays with energies above the GZK cutoff in a top-down approach. Here, we perform explicit calculations following such a suggestion by assuming that $\psi$ particles are produced nonrelativisticaly. In this way the energy transfer process from the inflaton field is more efficient, since only a very "fat" $\chi$ particle can decay into $\psi$ particles. The whole process can be schematically represented as

$$
\stackrel{g}{g \rightarrow} \stackrel{g}{\prime}^{\rightarrow} \psi
$$

where $g^{\prime}$ is the coupling constant of a Yukawa interaction $g^{\prime} \bar{\psi} \psi \chi$ added to the Lagrangian Eq. (1) to account for the interaction between $\psi$ and $\chi$.

From the Yukawa interaction term, the decay rate of $\chi$ bosons into $\psi$ fermions is

$$
\Gamma(\chi \rightarrow \bar{\psi} \psi)=\frac{g^{\prime 2} m_{\chi}^{\mathrm{eff}}}{16 \pi}\left[1-\left(\frac{2 m_{\psi}}{m_{\chi}^{\mathrm{eff}}}\right)^{2}\right]^{3 / 2} .
$$

Note that the above rate is not constant, since $m_{\chi}^{\text {eff }}$ defined by (9) is time-dependent, and $\chi$ particles tend to decay at large values of $\phi(t)$. This is the most interesting feature of the model: a great amount of energy can be transferred from $\phi$ to the stable fermions because the bosons $\chi$ decay when their variable masses are at a maximum value.

It is possible to obtain a relation between the couplings $g$ and $g^{\prime}$ if we take the interval $\Delta t=t_{f}-t_{i}$ around the maximum value of $\phi$ evolution for which $m_{\chi}^{\text {eff }}$ is large enough to allow the decay of $\chi$ particles into nonrelativistic $\psi$ fermions. Denoting by $n_{i}$ and $n_{f}$ the number densities of $\chi$ before and after the passage of $\phi$ through the local minimum of $V(\phi)$, respectively, we have

$$
\ln \left(\frac{n_{f}}{n_{i}}\right)=-\int_{t_{i}}^{t_{f}} \Gamma(t) d t .
$$

It is convenient to work with a more intuitive time variable, i.e., the number of oscillations

$$
N=\frac{m_{\phi} t}{2 \pi}
$$

of $\phi$. In such a case, the solution (4) for $\phi$ becomes

$$
\phi(N) \approx \frac{M_{P l}}{3} \frac{\sin (2 \pi N)}{2 \pi N} .
$$

This is a good parametrization from $N=0.25$ and later if we fix $N=0.5$ to be the instant when the inflaton field crosses the minimum of its potential for the first time. We will consider that the maximum momentum of $\psi$ particle to be its mass, that is, $p_{\psi} \leq m_{\psi}$. The maximum available energy for the creation of a $\psi$ pair is $g \Phi$, where $\Phi$ is the amplitude of $\phi(N)$. This determines the largest interval $\Delta N$ around the maximum amplitude of the $\phi$ field, $N \approx 0.72 \quad(\Phi$ $\approx 0.07 M_{P l}$ ), for which $m_{\chi}^{\text {eff }} \approx g \Phi$ is large enough to allow a pair creation. Such an interval is found to be $\Delta N=0.25$. Additionally, we require that approximately $90 \%$ of the $\chi$ particles decay during this interval. From Eq. (11) we obtain,

$$
\ln \left(\frac{10}{100}\right) \lesssim-\frac{1}{2^{3 / 2}} \times \frac{g^{\prime 2} g}{16 \pi} \int_{0.60}^{0.852} \frac{2 \pi}{m_{\phi}} \phi(N) d N,
$$

with $\phi(N)$ given by (13). Solving numerically the above integral, we obtain that

$$
g^{\prime 2} g \gtrsim 3 \times 10^{-3} .
$$

This upper limit is almost one order of magnitude larger than the Felder et al. estimate [15] $\left(g^{\prime 2} g \approx 5 \times 10^{-4}\right)$, since they did not consider the phase space factor in the expression for the decay rate (10). It is important to notice that the only arbitrariness in our assumptions is the fraction of remaining $\chi$ particles $(90 \%)$, but for reasonable choices (say $n_{f} / n_{i}$ between $1 / 2$ and 1/100) the constraints on $g$ and $g^{\prime}$ do not vary appreciably. All the other constraints are consequences of the assumption of a maximum momentum $p_{\psi} \leqslant m_{\psi}$ so that $\psi$ may be considered as nonrelativistic. Naturally, the limits on $g^{\prime 2} g$ would be even tighter if we had considered values for larger momenta. Therefore, we are being conservative in our estimates. We also verified that although one should assume the time dependence of the decay rate on the above calculations, it does not bring any important difference if compared to the estimate found in Ref. [15] where a constant decay rate, $\Gamma$, was used. This seems reasonable, since the integration interval for $N$ is taken to be around the maximum of $\phi(N)$ where the sine function does not vary significantly. Such care would be necessary if we were studying the production of relativistic particles.

Independently of the calculation details, our main goal in this work is to find the largest possible region of the parameter space of the FKL mechanism that is phenomenologically allowed by the available data. Assuming that the $\psi$ fermions are the metastable massive particles that we are looking for, we need to evaluate their present abundance supposing that they contribute to the energy density of the dark matter today. Since for each decaying $\chi$ a $\bar{\psi} \psi$ pair is created, we have to find the number density $n_{\chi}$ of the $\chi$ bosons for each oscillation of the inflaton. For the first oscillation, $n_{\chi}$ can be calculated from the solution of Eq. (7) about the minimum of $V(\phi)$, so that the total number of $\chi$ particles [15] is 


$$
n_{\chi}=\frac{1}{(2 \pi)^{3}} \int d^{3} k n_{k}=\frac{\left(g\left|\dot{\phi}_{0}\right|\right)^{3 / 2}}{8 \pi^{3}} \exp \left(-\frac{\pi m_{\chi}^{2}}{g\left|\dot{\phi}_{0}\right|}\right),
$$

and $\dot{\phi}_{0}=m_{\phi} \Phi / \pi$ is the field velocity near the minimum of the potential. The model describes the above production of $\chi$ particles, the boosting of their masses and their subsequent decay into $\psi$ wimpzillas with masses

$$
m_{\psi} \approx g \Phi \approx 0.07 g M_{P l} .
$$

In order to verify that most of the $\psi$ particles will be produced in the first oscillation of $\phi$, we compare the number density of produced $\chi$ particles in the second passage by the minimum of the $\phi$ potential, $n_{\chi}^{(2)}$, to the first one, $n_{\chi}^{(1)}$. By taking into account the dilution of $n_{\chi}^{(1)}$ due to the Universe expansion between the consecutive passages, it is found that (for details see the Appendix)

$$
\frac{n_{\chi}^{(2)}}{n_{\chi}^{(1)}}=\sqrt{2} \exp \left(\frac{-3 \pi^{2} \times 10^{6}}{g} \frac{m_{\chi}^{2}}{M_{P l}^{2}}\right) .
$$

Therefore, the exponential term could provide the desired suppression between the two first passages. In fact, it can be shown that the ratio between two consecutive passages tends to a constant prefactor multiplying the exponential, and, as long as the suppression is assured, particle production will be negligible for all subsequent oscillations. We will see in the next section that such suppression is verified, since for typical values of the parameters, say, $g=10^{-2}$ and $m_{\chi}$ $=10^{-4} M_{P l}, n_{\chi}^{(2)} \approx 10^{-26} n_{\chi}^{(1)}$ and hence it is reasonable to consider only the first passage in our calculations.

\section{IV. \& ABUNDANCE AND ULTRAHIGH ENERGY COSMIC RAYS}

If the $\psi$ particles are the superheavy relics that decay into the observed UHECR's, we can use the presumed cosmic ray flux associated to their decays [2] in order to estimate limits on the parameters of the model discussed above.

By accounting for the dilution of $\psi$ particles since their production until today, one may find the associated density parameter $\Omega_{\psi}\left(t_{0}\right)=\rho_{\psi}\left(t_{0}\right) / \rho_{c}$. In order to obtain an expression for this parameter, we have to consider three different moments in the history of the Universe: the production of $\psi$ particles $\left(t_{p}\right)$, the end of the reheating period $\left(t_{r h}\right)$, and today $\left(t_{0}\right)$. We can then write:

$$
\frac{\rho_{\psi}\left(t_{0}\right) T_{0}}{\rho_{R}\left(t_{0}\right)}=\frac{\rho_{\psi}\left(t_{r h}\right) T_{r h}}{\rho_{R}\left(t_{r h}\right)},
$$

where we assumed thermal equilibrium for the relativistic components and the fact that $\rho_{\psi}\left(t_{0}\right) a_{0}^{3}=\rho_{\psi}\left(t_{r h}\right) a_{r h}^{3}$. On the other hand we can suppose that the Universe will be reheated from an instant convertion of the remanescent inflaton density energy into relativistic particles, so that

$$
\frac{\rho_{\psi}\left(t_{r h}\right)}{\rho_{R}\left(t_{r h}\right)}=\frac{\rho_{\psi}\left(t_{r h}\right)}{\rho_{\phi}\left(t_{r h}\right)}=\frac{\rho_{\psi}\left(t_{p}\right)}{\rho_{\phi}\left(t_{p}\right)},
$$

where the last equality is obtained by considering that the $\psi$ and $\phi$ field coherent oscillations redshift as non relativistic matter. Note that we are supposing that only the $\psi$ particle production in the first passage is important [see expression (18)].

Finally we can substitute $\rho_{\phi}\left(t_{p}\right)=\left(3 M_{P l}^{2} / 8 \pi\right) H_{p}^{2}$ into (19) so that

$$
\Omega_{\psi}\left(T_{0}\right) h^{2}=\Omega_{R}\left(T_{0}\right) h^{2}\left(\frac{T_{r h}}{T_{0}}\right) \frac{8 \pi}{3} \frac{m_{\psi} n_{\psi}}{M_{P l}^{2} H_{p}^{2}},
$$

where $T_{0} \approx 2.7 \mathrm{~K}$ is the present cosmic microwave background (CMB) temperature, $h \equiv H /\left(100 \mathrm{~km} \mathrm{~s}^{-1} \mathrm{Mpc}^{-1}\right)$ and $\Omega_{R}\left(T_{0}\right) h^{2} \approx 4.3 \times 10^{-5}$ is the current radiation density parameter. The other parameters are $H_{p}^{2}=\left(8 \pi / 3 M_{P l}^{2}\right)\left(\rho_{\phi_{p}}\right)$ $=\left(8 \pi / 3 M_{P l}^{2}\right)\left(m_{\phi}^{2} \Phi^{2} / 2\right) \approx(4 \pi / 3) \times 10^{-14} M_{P l}^{2}$ and, given that $n_{\chi}=n_{\psi} / 2$ and for nonrelativistic fermions $m_{\chi}^{\text {eff }} \approx 2 m_{\psi}$, we find that $m_{\psi} n_{\psi} \approx m_{\chi}^{\text {eff }} n_{\chi}$, where $n_{\chi}$ is given by Eq. (16). Choosing $T_{r h}=10^{9} \mathrm{GeV}$, the above equation can be rewritten as

$$
\begin{aligned}
\Omega_{\psi}\left(T_{0}\right) h^{2} \approx & 4.72 \times 10^{18}\left(T_{r h} 10^{9} \mathrm{GeV}\left(\frac{2.7 \mathrm{~K}}{T_{0}}\right)\right. \\
& \times g^{3 / 2} \sqrt{\frac{m_{\chi}^{2}}{M_{P l}^{2}}+10^{-2} g^{2}} \\
& \times \exp \left(-\frac{\pi^{2} \times 10^{7}}{g} \frac{m_{\chi}^{2}}{M_{P l}^{2}}\right) .
\end{aligned}
$$

If $\psi$ wimpzillas are required to explain UHECR's, it is possible to obtain a relation between their abundance $\left(\Omega_{\psi}\right)$, their masses $\left(m_{\psi}\right)$, the lifetime of $\psi\left(\tau_{\psi}\right)$, and the UHECR's fluxes (for details see Refs. [2,19]). As we mentioned earlier, in order to obtain such relations it is necessary to adopt a model in which particles are produced from the $\psi$ decay. This topic is an issue by itself, and we will follow the usual assumption of extrapolating QCD mechanisms valid in the the quark-hadron fragmentation process for the higher energies considered here. As we will show later on, the use of a specific value for the flux will not alter the region of the allowed parameter space appreciably. Such a choice implies that photons dominate the primary spectrum by a factor of $\approx 6$ over protons [2]. This means that, if we consider the observable ultrahigh energy cosmic ray fluxes as due to extremely energetic photons resulting from the decay of $\psi$ particles, we can write

$$
\tau_{\psi}=3.16 \times 10^{18} f\left(\Omega_{\psi} h^{2}\right)\left(\frac{M_{P l}}{m_{\psi}}\right)^{1 / 2},
$$

where $f$ measures the clustering of $\psi$ particles inside the galactic halo. It is taken as 1 for a uniform distribution of superheavy WIMPs, but can be considered as approximately $10^{3}$ if they are concentrated in galactic halos (see [2]). We 




FIG. 1. The allowed parameter space $\left(m_{\chi}, g, g^{\prime}\right)$ for the production of wimpzillas in the FKL mechanism. For $m_{\chi}$ given in Planck mass units $\left(m_{\chi_{P l}}\right)$, the dark gray area stands for the allowed values of the parameters between $\Omega_{\psi} h^{2}=0.14$ (dashed line) and $\Omega_{\psi} h^{2}$ $=3.16 \times 10^{-12}\left(g \Phi / M_{P l}\right)^{1 / 2}$ (solid line). Unitarity constraints on $g^{\prime}$ and $g$ provide limits to the left and to the right in the allowed area in the figure. The application of these limits on the original FKL results [15], $g^{\prime 2} g \sim 5 \times 10^{-4}$, increases the allowed area by adding the light gray region.

will assume the latter case in what follows, i.e., $f \approx 10^{3}$, so that following limits on the parameters of the model can be established:

(1) if they constitute the whole of the dark matter (for $\left.\Omega_{\psi} \sim 0.3, h \sim 0.7[18]\right)$

$$
\Omega_{\psi}\left(T_{0}\right) h^{2} \sim 0.14
$$

(2) if they have a lifetime of the order of the age of the Universe

$$
\tau_{\psi} \approx 10^{10} \text { years } \rightarrow \Omega_{\psi}\left(T_{0}\right) h^{2} \approx 3.16 \times 10^{-12}\left(\frac{g \Phi}{M_{P l}}\right)^{1 / 2} .
$$

Therefore, the $\psi$ abundance obeys the limits 3.16 $\times 10^{-12}\left(g \Phi / M_{P l}\right)^{1 / 2} \leq \Omega_{\psi} h^{2} \leqq 0.14$. This imposes constraints on the $\chi$ bare mass according to (22):

$$
\begin{gathered}
10^{6} g \ln \left[4.72 \times 10^{19} g^{3 / 2}\left(\frac{m_{\chi}^{2}}{M_{P l}^{2}}+10^{-2} g^{2}\right)^{1 / 2}\right] \leqslant \frac{m_{\chi}^{2}}{M_{P l}^{2}} \\
\leqslant 10^{6} g \ln \left[1.49 \times 10^{30} g^{3 / 2}\left(\frac{m_{\chi}^{2}}{M_{P l}^{2}}+10^{-2} g^{2}\right)^{1 / 4}\right] .
\end{gathered}
$$

Such conditions define a parameter space which is convenient to assess the phenomenological viability of the FKL mechanism as a generator of $\psi$ non relativistic wimpzillas. This is possible because the number density of $\chi$ produced in the post-inflationary era depends on $g$ and $m_{\chi}$ and is related to $\Omega_{\psi}$, so that limits on $\Omega_{\psi}$ today restrict the possible values of such parameters. The analysis is summarized in Fig. 1, which can be understood as follows. The upper and lower limits of the gray area come from the substitution of Eqs. (24) and (25) in (22). We also have imposed unitarity constraints on Eq. (15) ( $g$ and $\left.g^{\prime} \lesssim 1\right)$, so that $3 \times 10^{-3} \lesssim g \lesssim 1$ (which comprises the upper right triangle in the figure) and

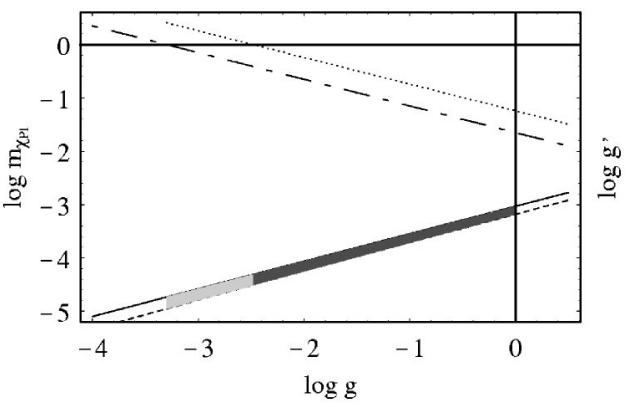

FIG. 2. The allowed parameter space of the previous figure is not substantially altered even if it is enlarged by an exaggerated and hypothetical lower limit for the abundance of $\psi\left(\Omega_{\psi} h^{2}=10^{-20}\right.$, solid line). All the other lines have the same meanings as in Fig. 1.

obtained the limits to the left (the minimum value for $g$ given the maximum possible value for $g^{\prime}$ ) and to the right (the maximum value for $g$ ) in the allowed (dark gray) area in the figure. For the sake of comparison, we also included the same analysis for the original FKL result, which corresponds to the right triangle limited by the dot-dashed line and to the widened area including the light gray region.

We see that the allowed region in the parameter space is rather constrained. Particularly, given the valid range for $g$, we find very high masses for $\psi$. From Eq. (17) the minimum $\psi$ mass that can be obtained through this model is $m_{\psi}$ $\sim 10^{15} \mathrm{GeV}$ (the upper limit being $m_{\psi} \sim 10^{18} \mathrm{GeV}$ ). This happens because the exponential suppression of the number density of $\chi$ particles created after the first $\phi$ field half oscillation, necessary to avoid parametric resonance [see Eq. (18)], is also present in the expression for $\Omega_{\psi}$ [see Eq. (22)]. Although the upper cosmological limit is quite strong given the most recent measurements, one may consider as a weak constraint the second astrophysical cosmic ray limit, as it is model dependent. However, even if we consider other classes of models to establish new lower limits on $\Omega_{\psi}$ the above results would not change significantly, because the parameters of the model are not very sensitive to variations on $\Omega_{\psi}$. Let us assume, for the sake of a comparison, an hypothetical value for the present $\psi$ abundance, say $\Omega_{\psi} h^{2}$ $\sim 10^{-20}$, so that the allowed region of the Fig. 1 is enlarged. The resulting parameter space is depicted in Fig. 2.

For the same reason, wide variations of the reheating temperature in Eq. (21) will not change the picture. For example, one would have to consider reheating temperatures about 20 orders of magnitude higher than the one assumed here to obtain a shift of only one order of magnitude on the allowed range of $m_{\chi_{P l}}$. Therefore, the resulting allowed area of the parameter space is relatively independent of particle physics details of the reheating and of the hypothetical top-down decay of $\psi$ particles in UHECR's.

\section{CONCLUSION}

We studied how to generate supermassive fermions that can explain the UHECR's in the context of a particle production mechanism suggested in Ref. [15]. We obtained the parameter space for which such a mechanism can take place 
and concluded that some fine tuning of parameters seems to be necessary. Additionally, the lower limit on the $\psi$ mass obtained, $m_{\psi} \geqslant 10^{15} \mathrm{GeV}$, is rather robust. A typical signature of this model would be an unforeseen rise in the flux at the highest energies end of the cosmic ray spectrum, which could be observed by the next generation of experiments, like the Pierre Auger Observatory. The cosmic ray spectrum must have a cutoff that is associated with the maximum energy possible for UHECR's and is independent of the GZK feature. If such a cutoff happens to be below $10^{15} \mathrm{GeV}$, this simplest version of the FKL mechanism should be discarded. On the other hand, it is this mechanism that can provide masses of such magnitude more naturally than any other topdown versions, so that if it is at all possible to measure such high energy cosmic rays and if no cutoff in the UHECR spectrum is observed by the next generation of experiments, then this model can become an attractive candidate. It is also interesting that, despite having perhaps too many free parameters, this model is rather constrained, and such a result is relatively insensitive to wide variations of the relevant cosmological parameters.

It is important to emphasize that we studied the production of nonrelativistic $\psi$ particles only, and the scenario can be made more complex by considering the production of $\psi$ particles that are relativistic at the preheating time but becomes nonrelativistic along the evolution of the Universe. In this case, the energy transfer from the inflaton field to other fields may be not very efficient and it would be necessary to consider the dilution/concentration of $\psi$ particles throughout the several phases that happened since inflation (coherent oscillations phase, radiation domination and matter domination) and the allowed parameter space may be widened.

\section{ACKNOWLEDGMENTS}

The authors are grateful to Hugo C. Reis for useful discussions. We also benefitted from suggestions and comments from Raul Abramo, Carlos O. Escobar, Alejandra Kandus, Gustavo Medina-Tanco, and Daniel Muller. This work was supported by FAPESP and CNPq.

\section{APPENDIX: THE EXPONENTIAL SUPPRESSION}

From (13), we can write the time derivative of $\phi(N)$ with respect to $N$ :

$$
\phi^{\prime}(N)=\frac{M_{P l}}{3} \frac{2 \pi \cos (2 \pi N)}{2 \pi N}-\frac{M_{P l}}{3} \frac{\sin (2 \pi N)}{2 \pi N^{2}} .
$$

By labelling each time that $\phi$ passes through the minimum of its potential as $N_{j}$, we have

$$
\phi_{j}^{\prime}=\frac{M_{P l}}{3} \frac{\cos \left(2 \pi N_{j}\right)}{N_{j}},
$$

that is, first passage:

$$
N_{1}=1 / 2 \rightarrow\left|\phi_{1}^{\prime}\right|=\frac{2}{3} M_{P l}
$$

second passage:

$$
N_{2}=2 / 2 \rightarrow\left|\phi_{2}^{\prime}\right|=\frac{1}{3} M_{P l}
$$

third passage:

$$
N_{3}=3 / 2 \rightarrow\left|\phi_{3}^{\prime}\right|=\frac{2}{9} M_{P l}
$$

$j$ th passage:

$$
N_{j}=j / 2 \rightarrow\left|\phi_{j}^{\prime}\right|=\left|\frac{M_{P l}}{3} \frac{\cos (j \pi)}{j / 2}\right|=\frac{M_{P l}}{3} \times \frac{2}{j} .
$$

From the general expression above, it is possible to obtain the ratio between the particle number density of $\chi$ particles produced in two consecutive bursts of $\chi$ generation so that we can evaluate the amount of suppression for each passage $j$. Writing the expression (16) for the total number density of $\chi$ particles produced in each passage in terms of the new definitions, we have

$$
\begin{aligned}
n_{\chi}^{(j)}\left(t_{j}\right)= & \frac{g^{3 / 2}}{8 \pi^{3}}\left(\frac{\left|\phi_{j}^{\prime}\right| m_{\phi}}{2 \pi}\right)^{3 / 2} \exp \left(-\frac{\pi m_{\chi}^{2}}{g\left|\phi_{j}^{\prime} m_{\phi} / 2 \pi\right|}\right) \\
\approx & \frac{g^{3 / 2}}{8 \pi^{3}}\left(\frac{M_{P l}}{3} \frac{2}{j} \frac{m_{\phi}}{2 \pi}\right)^{3 / 2} \\
& \times \exp \left(-\pi \frac{3}{M_{P l}} \frac{2 \pi}{m_{\phi}} \frac{j}{2} \frac{m_{\chi}^{2}}{g}\right) .
\end{aligned}
$$

Since the $a(t) \propto t^{2 / 3}$ along the coherent oscillation phase, we must compare the number density of particles produced at $t_{j}$ until $t_{j+1}$ by taking into account the dilution of $n_{j}$ at the ( $j$ $+1)$ th passage:

$$
\begin{array}{r}
n_{\chi}^{(j)}\left(t_{j}\right) a^{3}\left(t_{j}\right)=n_{\chi}^{(j)}\left(t_{j+1}\right) a^{3}\left(t_{j+1}\right) \\
\Rightarrow n_{\chi}^{(j)}\left(t_{j+1}\right)=n_{\chi}^{(j)}\left(t_{j}\right)\left(\frac{t_{j}}{t_{j+1}}\right)^{2} .
\end{array}
$$

However, from (12), $t_{j} / t_{j+1}=j / j+1$, so the ratio to be considered reads:

$$
\frac{n_{\chi}^{(j+1)}\left(t_{j+1}\right)}{n_{\chi}^{(j)}\left(t_{j+1}\right)}=\frac{n_{\chi}^{(j+1)}\left(t_{j+1}\right)}{n_{\chi}^{(j)}\left(t_{j}\right)}\left(\frac{j+1}{j}\right)^{2} .
$$


By using expression (A3) in the above relation, we finally find:

$$
\frac{n_{\chi}^{(j+1)}\left(t_{j+1}\right)}{n_{\chi}^{(j)}\left(t_{j+1}\right)} \approx\left(\frac{j+1}{j}\right)^{1 / 2} \exp \left(-\frac{3 \pi^{2} \times 10^{6}}{g} \frac{m_{\chi}^{2}}{M_{P l}^{2}}\right) .
$$

Since $\chi$ particles decay into two $\psi$ particles, the ratio above is also valid for $\psi$ particles. Thus, we conclude that the exponential suppression that has to be addressed in order to obey cosmological limits on the wimpzilla abundance (see Section IV) guarantees that we can consider to a good approximation only the $\chi$ particles produced in the first passage.
[1] M. Nagano and A.A. Watson, Rev. Mod. Phys. 72, 689 (2000). For more recent data from the AGASA Collaboration, see M. Takeda et al., Astropart. Phys. 19, 447 (2003). It is important to point out that such results have been questioned by data from the HiRes Collaboration, T. Abu-Zayyad et al., astro-ph/0208243. See also D. De Marco, P. Blasi, and A.V. Olinto, Astropart. Phys. 20, 53 (2003).

[2] P. Bhattacharjee and G. Sigl, Phys. Rep. 327, 109 (2000); R.J. Protheroe and R.W. Clay, Prog. Theor. Phys. Suppl. 151, 74 (2003).

[3] C.B. Finley and S. Westerhoff, Astropart. Phys. 21, 359 (2004).

[4] A.M. Hillas, Annu. Rev. Astron. Astrophys. 22, 425 (1984).

[5] K. Greisen, Phys. Rev. Lett. 16, 748 (1966); G.T. Zatsepin and V.A. Kuzmin, Pis'ma Zh. Éksp. Teor. Fiz. 4, 114 (1966) [JETP Lett. 4, 78 (1966)].

[6] F.A. Aharonian and J.W. Cronin, Phys. Rev. D 50, 1892 (1994).

[7] R.J. Protheroe and P.L. Biermann, Astropart. Phys. 6, 45 (1996); 7, 181(E) (1997).

[8] G. Domokos and S. Kovesi-Domokos, Phys. Rev. Lett. 82, 1366 (1999).

[9] V. Berezinsky, M. Kachelrie, and A. Vilenkin, Phys. Rev. D 79, 4302 (1997); V.A. Kuzmin and V.A. Rubakov, Phys. At. Nucl. 61, 1028 (1998).

[10] M. Birkel and S. Sarkar, Astropart. Phys. 9, 297 (1998); V. Berezinsky and M. Kachelriess, Phys. Rev. D 63, 034007 (2001); S. Sarkar and R. Toldra, Nucl. Phys. B621, 495 (2002); C. Barbot and M. Drees, Astropart. Phys. 20, 5 (2003).
[11] A. Vilenkin and E.P.S. Shellard, Cosmic Strings and Other Topological Defects (Cambridge University Press, Cambridge, England, 1994); M.B. Hindmarsh and T.W.B. Kibble, Rep. Prog. Phys. 55, 478 (1995).

[12] A. Barrau, Astropart. Phys. 12, 269 (2000).

[13] D.J.H. Chung, E.W. Kolb, and A. Riotto, Phys. Rev. D 59, 023501 (1999); V. Kuzmin and I. Tkachev, ibid. 59, 123006 (1999); D.J.H. Chung, P. Crotty, E.W. Kolb, and A. Riotto, ibid. 64, 043503 (2001); R. Allahverdi and M. Drees, Phys. Rev. Lett. 89, 091302 (2002).

[14] V.A. Kuzmin and I.I. Tkachev, Phys. Rep. 320, 199 (1999).

[15] G. Felder, L. Kofman, and A.D. Linde, Phys. Rev. D 59, 123523 (1999).

[16] A.H. Campos, H.C. Reis, and R. Rosenfeld, Phys. Lett. B 575, 151 (2003).

[17] M. Sami and V. Sahni, hep-th/0402086.

[18] W.L. Freedman and M.S. Turner, Rev. Mod. Phys. 75, 1433 (2003).

[19] A.H. Campos, L.L. Lengruber, H.C. Reis, R. Rosenfeld, and R. Sato, Mod. Phys. Lett. A A 17, 2179 (2002).

[20] A.D. Linde, Phys. Lett. 129B, 177 (1983).

[21] J.H. Traschen and R.H. Brandenberger, Phys. Rev. D 42, 2491 (1990); Y. Shtanov, J.H. Traschen, and R.H. Brandenberger, ibid. 51, 5438 (1995).

[22] L.A. Kofman, A.D. Linde, and A.A. Starobinsky, Phys. Rev. Lett. 73, 3195 (1994).

[23] L.A. Kofman, A.D. Linde, and A.A. Starobinsky, Phys. Rev. D 56, 3258 (1997).

[24] D.J.H. Chung, Phys. Rev. D 67, 083514 (2003). 